\title{
Experimental test of the strongly nonclassical character of a noisy squeezed single- photon state
}

Jezek, M.; Tipsmark, A.; Dong, R.; Fiurásek, J.; Mista, Jr., L. ; Filip, R.; Andersen, U. L.

Published in:

Physical Review A

Link to article, DOI:

10.1103/PhysRevA.86.043813

Publication date:

2012

Document Version

Publisher's PDF, also known as Version of record

Link back to DTU Orbit

Citation (APA):

Jezek, M., Tipsmark, A., Dong, R., Fiurásek, J., Mista, Jr., L., Filip, R., \& Andersen, U. L. (2012). Experimental test of the strongly nonclassical character of a noisy squeezed single-photon state. Physical Review A, 86(4), 043813. https://doi.org/10.1103/PhysRevA.86.043813

\section{General rights}

Copyright and moral rights for the publications made accessible in the public portal are retained by the authors and/or other copyright owners and it is a condition of accessing publications that users recognise and abide by the legal requirements associated with these rights.

- Users may download and print one copy of any publication from the public portal for the purpose of private study or research.

- You may not further distribute the material or use it for any profit-making activity or commercial gain

- You may freely distribute the URL identifying the publication in the public portal 


\title{
Experimental test of the strongly nonclassical character of a noisy squeezed single-photon state
}

\author{
M. Ježek, ${ }^{1,2}$ A. Tipsmark, ${ }^{2}$ R. Dong, ${ }^{2,3}$ J. Fiurášek, ${ }^{1}$ L. Mišta, Jr., ${ }^{1}$ R. Filip, ${ }^{1}$ and U. L. Andersen ${ }^{2}$ \\ ${ }^{1}$ Department of Optics, Palacký University, 17. listopadu 1192/12, CZ-771 46 Olomouc, Czech Republic \\ ${ }^{2}$ Department of Physics, Technical University of Denmark, Fysikvej, DK-2800 Kgs. Lyngby, Denmark \\ ${ }^{3}$ Quantum Frequency Standards Division, National Time Service Center (NTSC), Chinese Academy of Sciences, \\ 710600 Lintong, Shaanxi, China
}

(Received 11 July 2012; published 10 October 2012)

\begin{abstract}
We experimentally verify the quantum non-Gaussian character of a conditionally generated noisy squeezed single-photon state with a positive Wigner function. Employing an optimized witness based on probabilities of squeezed vacuum and squeezed single-photon states, we prove that the state cannot be expressed as a mixture of Gaussian states. In our experiment, the non-Gaussian state is generated by conditional subtraction of a single photon from a squeezed vacuum state. The state is probed with a homodyne detector and the witness is determined by averaging a suitable pattern function over the measured homodyne data. Our experimental results are in good agreement with a theoretical fit obtained from a simple yet realistic model of the experimental setup.
\end{abstract}

DOI: 10.1103/PhysRevA.86.043813

PACS number(s): 42.50.Dv, 03.65.Ta

\section{INTRODUCTION}

Quantum nonclassicality is a fundamental feature of quantum physics and a key resource in modern applications such as quantum information processing and quantum metrology. At the early stage of quantum optics, coherent states were identified as quantum states establishing a bridge between classical and quantum coherence theory [1]. Coherent states can be generated from vacuum by a classical coherent driving described by an interaction Hamiltonian linear in the annihilation $a$ and creation $a^{\dagger}$ operators. Coherent states provide a quantum description of classical coherent light waves, and their mixtures $\rho=\int P(\alpha)|\alpha\rangle\langle\alpha| d^{2} \alpha$ are therefore considered to be classical because they are described by a positivesemidefinite Glauber-Sudarshan function $P(\alpha)$ [1], which can be treated as a probability density of wave amplitudes. On the other hand, if $P(\alpha)$ cannot be interpreted as a probability density, the state becomes nonclassical from the point of view of coherence theory.

Operationally, nonclassical states cannot be prepared using only coherent states and passive linear optical elements. The lowest-order nonlinearity capable of producing a nonclassical state is described by interaction Hamiltonians quadratic in $a$ and $a^{\dagger}$. Such a quadratic nonlinearity can generate nonclassical squeezed states whose $P(\alpha)$ cannot be considered as an ordinary probability distribution. However, the squeezed states can still be described by a positive-definite Gaussian Wigner function $W(\alpha)[1]$.

In analogy to the set of mixtures of coherent states, we can introduce a set $\mathcal{G}$ of all Gaussian states and their mixtures, $\rho=\int P(\lambda) \rho_{G}(\lambda) d \lambda$, where $\rho_{G}(\lambda)$ denotes a Gaussian state, multi-index $\lambda$ labels all possible Gaussian states, and $P(\lambda)$ is a probability density. By definition, all states in $\mathcal{G}$ possess a positive Wigner function. Note that, according to the Hudson theorem, the Wigner function of any pure non-Gaussian state is negative at some point in phase space [2], and these states are thus highly nonclassical. On the other hand, a non-Gaussian Wigner function of a mixed state $\rho$ generally does not imply that the state is highly nonclassical, as this non-Gaussianity might arise solely from a non-Gaussian distribution, $P(\lambda)$, of states. This is an example of a classical non-Gaussianity. In contrast, we define quantum non-Gaussian states as all states which do not belong to $\mathcal{G}[3,4]$. The quantum non-Gaussian states are highly nonclassical because they cannot be prepared from thermal or coherent states using only Gaussian operations such as squeezing, and classical randomization. This means that some higher-order nonlinearity is necessarily involved in the state preparation. In view of the no-go theorems for entanglement distillation [5-7], quantum error correction [8], and quantum computing [9-11] with Gaussian states and operations, quantum non-Gaussian states, therefore, represent an enabling resource for continuous variable quantum information processing $[12,13]$.

It is therefore very important to develop reliable techniques for experimental identification of the quantum non-Gaussian states. Determining whether any given state is nonclassical is generally a very challenging task that may require an investigation of an infinite number of conditions [14]. Moreover, many experiments provide only partial information about the observed state. Fortunately, these difficulties can be overcome by formulating specific criteria that provide a sufficient condition for nonclassicality. Any such criterion can unambiguously verify nonclassicality of a class of quantum states, while for other states the result is inconclusive.

Recently, it has been shown that the quantum non-Gaussian character can be witnessed simply by determining the probabilities for the occurrence of vacuum and single-photon states $p_{0}=\langle 0|\rho| 0\rangle, p_{1}=\langle 1|\rho| 1\rangle$ [3]. This criterion allows us to conclusively certify the quantum non-Gaussian character of a large set of states, including those with positive Wigner functions. Simultaneously, it can be used to detect the presence of processes with higher than quadratic quantum nonlinearity.

Detection techniques typically register either particlelike or wavelike properties of quantum states. In optical settings where both types of detectors can be employed, a reliable direct measurement of the photon number is possible only for a low number of photons. When determining the photon number probabilities from coincidence measurements with realistic single-photon detectors that only distinguish the presence or absence of photons, we need to ensure that nonclassical features are not overestimated [4]. Alternatively, 
the probabilities $p_{0}$ and $p_{1}$ can be estimated indirectly by homodyne tomography [15-17]. A homodyne detector is used to measure rotated quadratures $x_{\theta}=\frac{1}{\sqrt{2}}\left(a e^{-i \theta}+a^{\dagger} e^{i \theta}\right)$, where $\theta$ is the relative phase between a strong coherent local oscillator and a signal beam, and the probabilities $p_{0}$ and $p_{1}$ are then reconstructed from the measured data by post-processing. In addition, coherent displacement or even squeezing can be applied to the experimental data so that we can determine $p_{0}$ and $p_{1}$ of a displaced and/or squeezed version of the original state only by data processing without performing these operations physically on the signal mode. This is a very useful technique that significantly broadens the applicability of the criterion. The highly nonclassical character of many states may be masked by a Gaussian envelope and the knowledge of $p_{0}$ and $p_{1}$ may not suffice for the identification of quantum non-Gaussian character of those states. The above technique can remove this Gaussian veil and greatly enhance the power of the criterion.

In this paper, we experimentally certify the quantum nonGaussianity of a conditionally generated squeezed singlephoton state [18-25] whose Wigner function is positive at the origin of phase space due to noise. The state is prepared by conditionally subtracting a single photon from a squeezed vacuum state, and it is probed with a homodyne detector. Probabilities $p_{0}$ and $p_{1}$ are determined by two different estimation methods, namely linear reconstruction based on pattern functions [26-30] and nonlinear maximum likelihood estimation [31-33]. To optimally witness the quantum non-Gaussian character of the measured state, we apply a suitable antisqueezing operation to the experimental data. We conclusively prove the quantum non-Gaussian character of the prepared state with confidence of 2.4 standard deviations. The experimental results are successfully fitted with a standard theoretical model of photon subtraction from squeezed vacuum.

The rest of the paper is organized as follows. The criterion allowing identification of quantum non-Gaussian states is discussed in Sec. II. The experimental setup for the generation of photon-subtracted squeezed states is described in Sec. III, and a theoretical model of the setup is presented in Sec. IV. The reconstruction of photon number distribution from experimental data by pattern functions and maximum likelihood estimation is discussed in Sec. V. Experimental results are presented in Sec. VI. Finally, Sec. VII contains brief conclusions.

\section{QUANTUM NON-GAUSSIAN STATES}

A simple and powerful criterion for practical identification of quantum non-Gaussian states was recently proposed in Ref. [3]. This criterion is based on a determination of the maximum probability of a single-photon state, $p_{1, G}$, that can be achieved by Gaussian states and their mixtures for a fixed probability of vacuum $p_{0}$. If $p_{1}$ exceeds this bound, then the state is quantum non-Gaussian. In fact, it suffices to maximize $p_{1}$ over pure squeezed coherent states that form extremal points of $\mathcal{G}$. This optimization yields a parametric description of the dependence of $p_{1, G}$ on $p_{0}$ [4],

$$
p_{0}=\frac{e^{-e^{r} \sinh (r)}}{\cosh (r)}, \quad p_{1, G}=\frac{e^{4 r}-1}{4} \frac{e^{-e^{r} \sinh (r)}}{\cosh ^{3}(r)},
$$

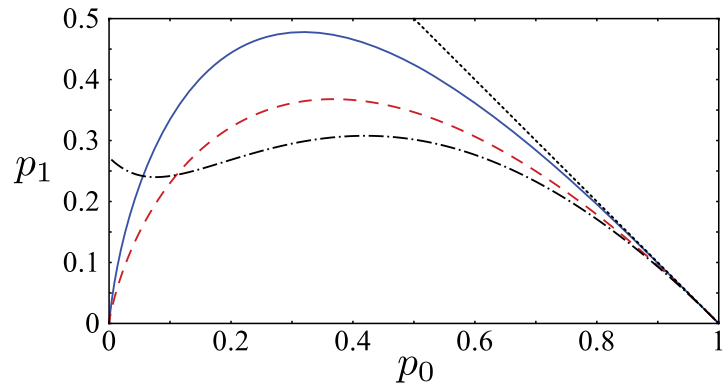

FIG. 1. (Color online) The maximum $p_{1}$ achievable by Gaussian states and their mixtures is plotted as a function of $p_{0}$ (solid blue line). The red dashed line represents the nonclassicality boundary given by Eq. (5) and the dotted black line indicates the ultimate physical boundary $p_{0}+p_{1}=1$. The dot-dashed black line represents a trajectory in the $\left(p_{0}, p_{1}\right)$ plane of a squeezed single-photon state $(r=1)$ subject to varying losses. The leftmost point corresponds to no losses $(\eta=1)$ while the rightmost point corresponds to complete losses $(\eta=0)$.

where $r \in[0, \infty)$. The boundary curve (1) is plotted in Fig. 1. Points lying above this curve can only be obtained for states that do not belong to $\mathcal{G}$. Interestingly, the class of quantum non-Gaussian states is much larger than the class of states with a negative Wigner function. For example, a mixture of vacuum and single-photon states with a dominant vacuum contribution, $\rho=p|0\rangle\langle 0|+(1-p)| 1\rangle\langle 1|, p>\frac{1}{2}$, has a positive Wigner function, yet it can be shown that it cannot be expressed as a mixture of Gaussian states for any $p>0$ [3].

Due to the convex structure of the set $\mathcal{G}$, we can construct a witness of the state's quantum non-Gaussian character. The witness is defined as a linear combination of $p_{0}$ and $p_{1}$,

$$
W(a)=a p_{0}+p_{1},
$$

where $a<1$ is a parameter specifying the witness [34]. The maximum value of $W(a)$ over $\mathcal{G}$ can be found by inserting formulas (1) into Eq. (2) and maximizing $W(a)$ over $r$. After some algebra, one obtains

$$
W_{G}(a)=\left(a+\frac{e^{4 r_{0}}-1}{4 \cosh ^{2}\left(r_{0}\right)}\right) \frac{e^{-e^{r_{0}} \sinh \left(r_{0}\right)}}{\cosh \left(r_{0}\right)},
$$

where

$$
r_{0}=\frac{1}{2} \ln \frac{3-a+\sqrt{a^{2}-10 a+9}}{2}
$$

is the optimal $r$ for a given $a$. If $W(a)>W_{G}(a)$, then the state is quantum non-Gaussian. Each optimal witness can be represented by a straight line $a p_{0}+p_{1}=W_{G}(a)$ which is tangent to the boundary curve (1).

In a similar fashion, we can also investigate whether the state is nonclassical in the sense that it cannot be expressed as a mixture of coherent states $|\alpha\rangle$. In this case, the maximum $p_{1}$ achievable for a fixed $p_{0}$ is specified by Poissonian statistics and no further optimization is necessary [35],

$$
p_{0}=e^{-\bar{n}}, \quad p_{1}=\bar{n} e^{-\bar{n}},
$$

where $\bar{n} \in[0, \infty)$ is the mean photon number. The boundary (5) is also plotted in Fig. 1 as a dashed line. By analogy, we can use $W(a)$ also as the nonclassicality witness. The bound 
achievable by mixtures of coherent states reads $W_{\mathrm{cl}}=e^{a-1}$ [35].

In this work, we are interested in the quantum nonGaussianity of approximate squeezed single-photon states obtained by photon subtraction from a squeezed vacuum [18-25]. In the ideal case of perfect single-photon subtraction from pure squeezed vacuum, we would obtain a pure squeezed single-photon state $|\psi(r)\rangle=S(r)|1\rangle$, where the squeezing operation reads

$$
S(r)=e^{-i \frac{r}{2}(x p+p x)},
$$

$r$ is the squeezing constant, and $x$ and $p$ denote conjugate quadrature operators satisfying canonical commutation relations, $[x, p]=i$. The witness $W(a)$ identifies the pure state $|\psi(r)\rangle$ as quantum non-Gaussian for any amount of squeezing, because it is a superposition of odd Fock states and $p_{0}=0$ while $p_{1}>0$. As a more realistic case, let us next consider a mixed state obtained by sending $|\psi(r)\rangle$ through a lossy channel $\mathcal{L}$ with transmittance $\eta$. In Fig. 1 , we plot the trajectory of points $p_{0}, p_{1}$ generated by varying the transmittance $\eta$ for a fixed amount of initial squeezing $r$. We can see that the curve enters the region of Gaussian mixtures, so for high enough losses we can no longer identify the state as quantum non-Gaussian or as nonclassical. There exists a threshold transmittance $\eta_{\text {th }}$ for which our criterion reveals the quantum non-Gaussian character of the state. The dependence of $\eta_{\text {th }}$ on the initial squeezing $r$ is plotted in Fig. 2(a).

Since we are dealing with a squeezed state, we can intuitively expect that a witness based on Fock state probabilities will not be optimal. We can significantly improve the performance of our witness if we first extract the highly nonclassical core of the state [36] by antisqueezing the state, $\rho_{S}=S^{\dagger}(s) \rho S(s)$. Note that the squeezing is a Gaussian operation that maps mixtures of Gaussian states onto mixtures of Gaussian states. Therefore, if $\rho_{S}$ is quantum non-Gaussian, then $\rho$ is also quantum non-Gaussian. Equivalently, we can introduce a generalized witness,

$$
W(a, s)=a p_{0}(s)+p_{1}(s),
$$

where $p_{n}(s)=\left\langle n\left|S^{\dagger}(s) \rho S(s)\right| n\right\rangle$ are diagonal density matrix elements in the basis of squeezed Fock states $S(s)|n\rangle$.
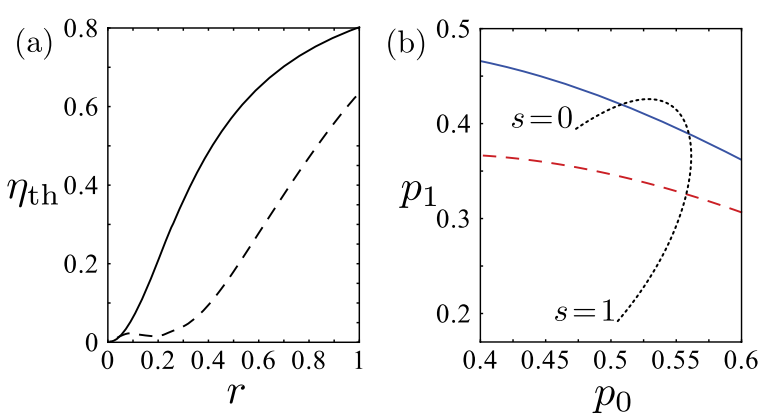

FIG. 2. (Color online) (a) Threshold transmittances $\eta_{\text {th }}$ (solid line) and $\eta_{\mathrm{th}, s}$ (dashed line) corresponding to witnesses $W(a)$ and $W(a, s)$, respectively, are plotted as a function of squeezing constant. (b) A $\left(p_{0}, p_{1}\right)$ plane trajectory of a lossy squeezed single-photon state $(r=0.5, \eta=0.4)$ subject to antisqueezing operation with a variable degree of antisqueezing $s$ (dotted black line). The blue solid line and red dashed line have the same meaning as in Fig. 1.
The antisqueezing partly compensates for the initial squeezing and makes the state closer to a mixture of single-photon and vacuum states for which the witness (2) is very powerful. Note that since the lossy channel and squeezing do not commute, $S \mathcal{L}(\rho) S^{\dagger} \neq \mathcal{L}\left(S \rho S^{\dagger}\right)$, the antisqueezing of the output mixed state is not fully equivalent to reducing the initial squeezing $r$. In Fig. 2(b), we plot a typical trajectory of $p_{0}, p_{1}$ pairs when the antisqueezing $s$ is varied. We can see that for a suitably chosen $s$, we can detect the quantum non-Gaussian character of the state, although without antisqueezing (corresponding to $s=0$ ) our witness fails. For comparison, we plot in Fig. 2(a) the threshold transmittance $\eta_{\text {th,s }}$ above which the witness $W(a, s)$ detects the quantum non-Gaussian character of the considered state. We can see that $\eta_{\text {th }, s}<\eta_{\text {th }}$, and this gap is a clear indication of the enhanced power and increased applicability of the witness $W(a, s)$. Note that in addition to antisqueezing, we could also coherently displace the state to remove any coherent component. However, this is not necessary for our present purposes because the states studied in this paper do not contain any coherent component.

\section{EXPERIMENTAL SETUP}

The setup used for the experimental test of the nonclassical character of photon-subtracted squeezed states [25] is shown in Fig. 3. A cavity-dumped Ti:sapphire laser (Tiger-PS, TimeBandwidth Products) produces 5-ps-long pulses with a maximum energy of $50 \mathrm{~nJ}$, a repetition rate of $815 \mathrm{kHz}$, and a central wavelength of $830 \mathrm{~nm}$. The laser pulses are up-converted in the process of second harmonic generation (SHG) using a 3-mm-thick periodically poled crystal of potassium titanyl phosphate (PPKTP1). The remaining radiation at $830 \mathrm{~nm}$ is removed by a set of dichroic mirrors (DM). The frequencydoubled pulses are used as a pump in a similar crystal (PPKTP2), phase-matched for collinear and fully degenerate parametric generation (OPA). It produces a squeezed vacuum with squeezing strength tunable from 0 to $3.5 \mathrm{~dB}$.

The generated squeezed light impinges onto an asymmetric beam splitter (ABS) which reflects $7.7 \pm 0.3 \%$ of the signal to a single-photon detection setup. It consists of a narrowband Fabry-Pérot filter (FP, FWHM = $0.04 \mathrm{~nm}$ ) and a single-mode fiber (SMF) that guides the signal to an avalanche photodiode (APD) operated in Geiger mode (SPCM-AQR-14, PerkinElmer). We estimate the total quantum efficiency of the filtering and subsequent detection process to be approximately $8 \pm 1 \%$. The uncertainty of the efficiency is quite high, particularly due to the uncertainty of the detection probability of the APD. The signal transmitted by the ABS is mixed with a

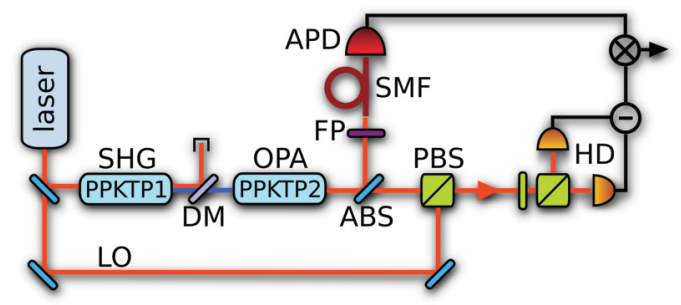

FIG. 3. (Color online) Experimental setup used to generate photon-subtracted squeezed states. 
local oscillator (LO) using polarizing beam splitters (PBS) and a half-wave plate (HWP), and detected by means of a pair of photodiodes (S3883, Hamamatsu). The difference of the photocurrents is generated and the resulting current is amplified by a charge-sensitive amplifier and finally fed into an oscilloscope working in the memory-segmentation regime. The acquisition is triggered by a detection event of APD and a synchronization pulse from the laser, which suppresses the effect of electronic dark counts (effectively below $3 \mathrm{~Hz}$ ). The homodyne detector (HD) efficiency of $80 \pm 3 \%$ is limited mainly by the efficiency of the photodiodes themselves (94\%), the mode matching of the signal and the LO (95\%), and the transmittance of passive optical elements in the signal path $(95 \%)$.

\section{THEORETICAL MODEL}

To compare the experimental results with theoretical predictions, we employ a standard model of squeezed singlephoton state preparation [37-39]. An equivalent scheme of the experimental setup is shown in Fig. 4, and our goal is to determine the probabilities $p_{0}$ and $p_{1}$ as functions of model parameters $V_{x}, V_{p}, T, \eta, \eta_{H}, n_{\mathrm{th}}$, and $Q$. Our derivation is based on phase-space representation and a Gaussian-state formalism because the non-Gaussian quantum state prepared by photon subtraction can be expressed as a weighted difference of two Gaussian states [37]. Any Gaussian state is fully characterized by first and second moments of quadrature operators, namely by covariance matrix $\gamma$ and the vector of coherent displacements $d$. Let us consider an $N$-mode system, and let $x_{j}$ and $p_{j}$ denote the amplitude and phase quadrature operators of mode $j$ satisfying the canonical commutation relations $\left[x_{j}, p_{k}\right]=i \delta_{j k}$. Upon collecting all quadrature operators into a vector $r=\left(x_{1}, p_{1}, \ldots, x_{N}, p_{N}\right)$, we can write $d=\langle r\rangle$ and $\gamma_{j k}=\left\langle\left\{\Delta r_{j}, \Delta r_{k}\right\}\right\rangle$, where $\{$,$\} denotes the anticommutator and$ $\Delta r_{j}=r_{j}-\left\langle r_{j}\right\rangle$. Coherent displacements of states involved in the model vanish, $d=0$, so the states are fully specified by their covariance matrices.

We assume that the input signal mode $A_{\text {in }}$ is prepared in a generally mixed squeezed vacuum state $\rho_{\text {in }}$ described

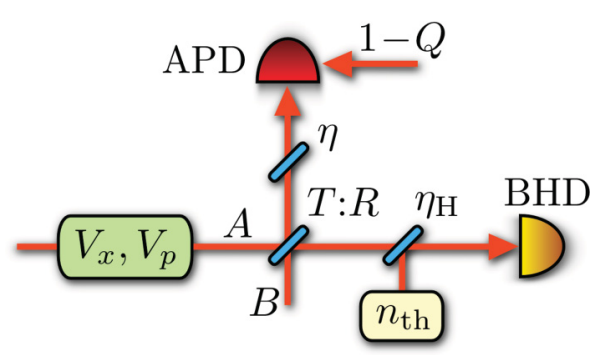

FIG. 4. (Color online) Equivalent theoretical model of the experimental setup. $V_{x}$ and $V_{p}$ denote variances of amplitude and phase quadratures of the input mixed squeezed vacuum state, and $T$ is the transmittance of the tap-off beam splitter. Inefficient single-photon (homodyne) detection is modeled as a sequence of a beam splitter with transmittance $\eta\left(\eta_{H}\right)$ followed by a perfect detector. Thermal photons with mean number $n_{\text {th }}$ can be injected into the signal beam which models electronic noise in the homodyne detector BHD. The single-photon detector APD can be triggered by dark counts or photons not coming from the signal mode which occurs with probability $1-Q$. by a diagonal covariance matrix $(\mathrm{CM}) \gamma_{A_{\text {in }}}=\operatorname{diag}\left(2 V_{x}, 2 V_{p}\right)$, where

$$
V_{x}=\left\langle\left(\Delta x_{A_{\text {in }}}\right)^{2}\right\rangle, \quad V_{p}=\left\langle\left(\Delta p_{A_{\text {in }}}\right)^{2}\right\rangle
$$

denote the variances of squeezed and antisqueezed quadratures, respectively, and $V_{x} V_{p} \geqslant \frac{1}{4}$. A small fraction of light is then tapped off by a strongly unbalanced beam splitter with intensity transmittance $T$ and reflectance $R=1-T \ll 1$ whose auxiliary input port $B$ is in the vacuum state. The state of modes $A$ and $B$ at the output of the beam splitter is Gaussian [40], and its Wigner function reads

$$
W_{A B}(\xi)=\frac{1}{\pi^{2} \sqrt{\operatorname{det} \gamma_{A B}}} e^{-\xi^{T} \gamma_{A B}^{-1} \xi}
$$

Here $\xi=\left(x_{A}, p_{A}, x_{B}, p_{B}\right)^{T}$ is the $c$-number phase-space coordinate vector, and the two-mode covariance matrix $\gamma_{A B}$ is given by

$$
\gamma_{A B}=\left(\begin{array}{cc}
T \gamma_{A_{\text {in }}}+R \gamma_{B_{\text {in }}} & \sqrt{R T}\left(\gamma_{A_{\text {in }}}-\gamma_{B_{\text {in }}}\right) \\
\sqrt{R T}\left(\gamma_{A_{\text {in }}}-\gamma_{B_{\text {in }}}\right) & R \gamma_{A_{\text {in }}}+T \gamma_{B_{\text {in }}}
\end{array}\right),
$$

where $\gamma_{B_{\text {in }}}=\mathbb{1}$ is the covariance matrix of vacuum. It is convenient to model inefficient single-photon detection as a combination of a lossy channel with transmittance $\eta$ followed by a perfect detector with unit efficiency. Similarly, we can model homodyne detection with efficiency $\eta_{H}$ and background noise $n_{\text {th }}$ by a sequence of a lossy channel with added thermal noise followed by a perfect homodyne detector. The two-mode covariance matrix that accounts for detection inefficiency and noise can be expressed as follows:

$$
\gamma_{A B}^{\prime}=M \gamma_{A B} M^{T}+G
$$

where

$$
M=\left(\begin{array}{cccc}
\sqrt{\eta_{H}} & 0 & 0 & 0 \\
0 & \sqrt{\eta_{H}} & 0 & 0 \\
0 & 0 & \sqrt{\eta} & 0 \\
0 & 0 & 0 & \sqrt{\eta}
\end{array}\right)
$$

and

$$
G=\left(\begin{array}{cccc}
1-\eta_{H}+2 n_{\mathrm{th}} & 0 & 0 & 0 \\
0 & 1-\eta_{H}+2 n_{\mathrm{th}} & 0 & 0 \\
0 & 0 & 1-\eta & 0 \\
0 & 0 & 0 & 1-\eta
\end{array}\right) .
$$

The on/off detector placed on mode $B$ can be described by projectors onto vacuum and the rest of the Hilbert space, respectively, as $\Pi_{0}=|0\rangle\langle 0|$ (no click) and $\Pi_{1}=\mathbb{1}-\Pi_{0}$ (click). If a click of the detector occurs, the state of mode $A$ collapses into the output state,

$$
\rho_{\text {out }}=\frac{\operatorname{Tr}_{B}\left(\mathbb{1}_{A} \otimes \Pi_{1, B} \rho_{A B}\right)}{\operatorname{Tr}\left(\mathbb{1}_{A} \otimes \Pi_{1, B} \rho_{A B}\right)}=\frac{1}{1-\mathcal{P}_{0}}\left(\rho^{(\mathrm{red})}-\mathcal{P}_{0} \rho^{(0)}\right),
$$

where $\rho_{A B}$ is the joint state of modes $A$ and $B$ in front of the detectors, $\rho^{\text {(red) }}=\operatorname{Tr}_{B}\left(\rho_{A B}\right)$ is the reduced state of the output mode $A, \mathcal{P}_{0}=\operatorname{Tr}\left(\mathbb{1}_{A} \otimes \Pi_{0, B} \rho_{A B}\right)$ is the probability of 
no click, and $\rho^{(0)}=\operatorname{Tr}_{A}\left(\mathbb{1}_{A} \otimes \Pi_{0, B} \rho_{A B}\right) / \mathcal{P}_{0}$ is the conditional state of mode $A$ corresponding to no click of the detector.

The output state can be most easily calculated using the formalism of Wigner functions because the POVM element $\Pi_{0}$ is a projector onto vacuum that possesses a Gaussian Wigner function. The Wigner function of the output state (14) can be written as a difference of two Gaussian Wigner functions centered on the origin,

$$
W_{\text {out }}\left(\xi_{A}\right)=\frac{1}{\pi\left(1-\mathcal{P}_{0}\right)}\left[\frac{e^{-\xi_{A}^{T} \gamma_{\mathrm{I}}^{-1} \xi_{A}}}{\sqrt{\operatorname{det} \gamma_{\mathrm{I}}}}-\mathcal{P}_{0} \frac{e^{-\xi_{A}^{T} \gamma_{0}^{-1} \xi_{A}}}{\sqrt{\operatorname{det} \gamma_{0}}}\right]
$$

Here $\xi_{A}=\left(x_{A}, p_{A}\right)^{T}$. To express the covariance matrices appearing in Eq. (15), we split the two-mode covariance matrix $\gamma_{A B}^{\prime}$ into single-mode blocks,

$$
\gamma_{A B}^{\prime}=\left(\begin{array}{cc}
\Gamma_{A} & \Gamma_{C} \\
\Gamma_{C}^{T} & \Gamma_{B}
\end{array}\right) .
$$

The covariance matrices read $\gamma_{I}=\Gamma_{A}$ and $\gamma_{0}=\Gamma_{A}-$ $\Gamma_{C}\left(\Gamma_{B}+\mathbb{1}\right)^{-1} \Gamma_{C}^{T}[6]$, and for the probability of no click we have

$$
\mathcal{P}_{0}=\frac{2}{\sqrt{\operatorname{det}\left(\Gamma_{B}+\mathbb{1}\right)}} .
$$

Despite heavy spatial and spectral filtering, the singlephoton detector APD can sometimes be triggered by photons from other modes than the mode which is subsequently observed by a homodyne detector. Such false triggers can also include dark counts caused by thermal fluctuations or other effects. We can define an effective mode overlap $Q$ as a probability that a click from the APD is caused by photons subtracted from the right mode. If the APD is triggered by a photon coming from some other mode, then the output state in mode $\mathrm{A}$ is prepared in a Gaussian state $\rho^{\text {(red) }}$ with zero mean and covariance matrix $\gamma_{I}$. The overall state in mode $\mathrm{A}$ is then a mixture of state (14) with probability $Q$ and a Gaussian state $\rho^{\text {(red) }}$ with probability $1-Q$. The Wigner function of the resulting state preserves the form $(15)$, but $\mathcal{P}_{0}$ is replaced with

$$
\mathcal{P}_{0}^{\prime}=\frac{Q \mathcal{P}_{0}}{1-\mathcal{P}_{0}(1-Q)} .
$$

Antisqueezing operation on the output state transforms the covariance matrix of each constituent Gaussian component according to $\gamma_{j} \rightarrow S \gamma_{j} S^{T}$, where $S=\operatorname{diag}\left(e^{-s}, e^{s}\right)$.

To evaluate the witness $W(a)$, we need to calculate the probabilities $p_{0}$ and $p_{1}$ of finding the output state (15) in the vacuum state and the single-photon Fock state, respectively. The probabilities can be derived from the overlap formula,

$$
p_{k}=2 \pi \int_{-\infty}^{\infty} \int_{-\infty}^{\infty} W_{\text {out }}\left(\xi_{A}\right) W_{|k\rangle}\left(\xi_{A}\right) d^{2} \xi_{A},
$$

where

$$
W_{|k\rangle}\left(\xi_{A}\right)=\frac{1}{\pi}\left[2 k \xi_{A}^{T} \xi_{A}+(-1)^{k}\right] \exp \left(-\xi_{A}^{T} \xi_{A}\right)
$$

are Wigner functions of vacuum $(k=0)$ and the single-photon Fock state $(k=1)$, respectively. Performing integration in Eq. (19), we arrive at the probabilities $p_{0}$ and $p_{1}$ in the following form:

$$
\begin{aligned}
& p_{0}=\frac{2}{1-\mathcal{P}_{0}^{\prime}}\left[\frac{1}{\sqrt{\operatorname{det}\left(\gamma_{I}+\mathbb{1}\right)}}-\frac{\mathcal{P}_{0}^{\prime}}{\sqrt{\operatorname{det}\left(\gamma_{0}+\mathbb{1}\right)}}\right], \\
& p_{1}=\frac{2}{1-\mathcal{P}_{0}^{\prime}}\left\{\frac{\operatorname{det} \gamma_{I}-1}{\left[\operatorname{det}\left(\gamma_{I}+\mathbb{1}\right)\right]^{\frac{3}{2}}}-\frac{\mathcal{P}_{0}^{\prime}\left(\operatorname{det} \gamma_{0}-1\right)}{\left[\operatorname{det}\left(\gamma_{0}+\mathbb{1}\right)\right]^{\frac{3}{2}}}\right\} .
\end{aligned}
$$

In Sec. VI, we will use these formulas to find the best theoretical fit to the experimental data.

\section{WITNESS ESTIMATION}

In this section, we describe the estimation techniques that were used for determination of the probabilities $p_{0}(s)$ and $p_{1}(s)$ from the experimental data. As a main tool, we utilize the pattern functions that provide unbiased linear estimators of $p_{j}(s)$ and allow for straightforward estimation of error bars, which is particularly important in the present case. For the sake of comparison, we also perform a maximum likelihood estimation of $p_{n}(s)$.

\section{A. Pattern functions}

A homodyne detector measures the probability distribution $w\left(x_{\theta} ; \theta\right)$ of a rotated quadrature operator $x_{\theta}=x \cos \theta+$ $p \sin \theta$, where $\theta$ is the relative phase between the local oscillator and the signal beam $[16,17]$. If the measurement is performed for values of $\theta$ spanning the whole interval $\theta \in$ $[0, \pi]$, then the measurement is tomographically complete and any element of the density matrix $\rho$ can be determined from the recorded data. We are interested in an estimation of diagonal density matrix elements in the Fock basis, i.e., photon number probabilities $p_{0}$ and $p_{1}$. A particularly straightforward method is to determine estimates of $p_{n}$ by averaging appropriate pattern functions $f_{n}\left(x_{\theta}\right)$ over the sampled quadrature statistics [26-30],

$$
p_{n}=\frac{1}{\pi} \int_{0}^{\pi} \int_{-\infty}^{\infty} w\left(x_{\theta} ; \theta\right) f_{n}\left(x_{\theta}\right) d x_{\theta} d \theta .
$$

Note that the pattern functions $f_{n}$ do not depend on the phase $\theta$. For vacuum and single-photon probabilities, we explicitly have [27-29]

$$
\begin{aligned}
& f_{0}(x)=2-2 \sqrt{\pi} x e^{-x^{2}} \operatorname{erfi}(x), \\
& f_{1}(x)=2\left(2 x^{2}-1\right)+4 \sqrt{\pi} x\left(1-x^{2}\right) e^{-x^{2}} \operatorname{erfi}(x),
\end{aligned}
$$

where $\operatorname{erfi}(x)$ denotes the error function of an imaginary argument. The functions (22) are plotted in Fig. 5, and we

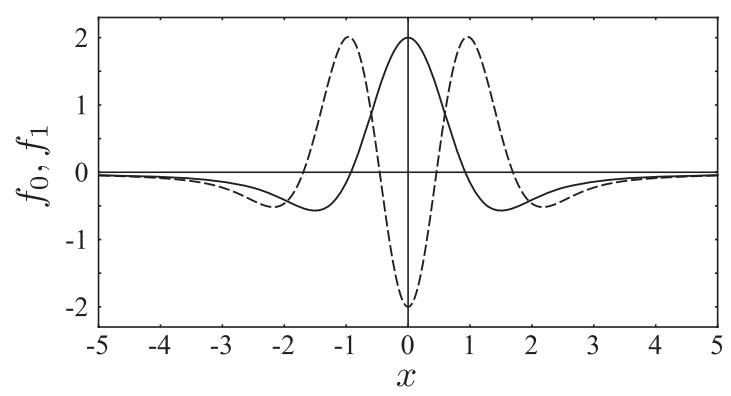

FIG. 5. Pattern functions $f_{0}(x)$ (solid line) and $f_{1}(x)$ (dashed line). 
can see that they are bounded and asymptotically approach 0 in the limit of large $|x|$. The pattern function for an estimation of witness $W(a)$ can be obtained as an appropriate linear combination of the two functions (22), $f_{W}=a f_{0}+f_{1}$.

Direct reconstruction based on pattern functions is an appealing option in situations in which we need to determine just some particular property of the state, such as the witness $W(a)$, and we do not want to reconstruct the whole state. In comparison with more sophisticated statistical reconstruction methods such as maximum likelihood estimation, the linear estimation is much faster and the statistical uncertainty of any estimated quantity can be easily determined [41] without calculating the Fisher matrix or performing complicated Monte Carlo simulations. To see this, let us assume that the quadratures were measured for $K$ different values of the phase shift $\theta_{k}=\frac{k}{K} \pi, k=1, \ldots, K$. In the actual experiment, the phase is approximately linearly modulated in time and is then fitted and binned into $K=40$ discrete equidistant values in the $[0, \pi]$ interval, so the following treatment is applicable to our data. Let $M_{k}$ denote the number of quadrature measurements for phase $\theta_{k}$ and let $X_{k, m}$ denote measurement outcomes for this setting, $m=1, \ldots, M_{k}$. In experimental data processing, the integral (21) is replaced with a finite sum,

$$
p_{n}=\frac{1}{K} \sum_{k=1}^{K} \frac{1}{M_{k}} \sum_{m=1}^{M_{k}} f_{n}\left(X_{k, m}\right)
$$

The inner sum represents averaging of the pattern function $f_{n}$ over sampled quadrature statistics for a fixed $\theta_{k}$, while the outer sum represents averaging over the phase $\theta$. If the number of quadrature samples $M_{k}$ is not a constant, then measurement results obtained for different values of $\theta$ have different weight in Eq. (23).

Statistical uncertainty of estimated $p_{n}$ can be quantified by its variance $V\left(p_{n}\right)=\left\langle p_{n}^{2}\right\rangle-\left\langle p_{n}\right\rangle^{2}$. Since $X_{k, m}$ are independent random variables, we find that

$$
V\left(p_{n}\right)=\frac{1}{K^{2}} \sum_{k=1}^{K} V_{k}\left(f_{n}\right)
$$

where

$$
V_{k}\left(f_{n}\right)=\frac{1}{M_{k}^{2}} \sum_{m=1}^{M_{k}}\left[\left\langle f_{n}^{2}\left(X_{k, m}\right)\right\rangle-\left\langle f_{n}\left(X_{k, m}\right)\right\rangle^{2}\right] .
$$

All quadrature measurement outcomes $X_{k, m}$ obtained for a fixed $k$ exhibit the same statistical distribution, therefore the statistical averages appearing in Eq. (25) can be estimated from the measured data, and we obtain

$$
V_{k}\left(f_{n}\right)=\frac{1}{M_{k}^{2}} \sum_{m=1}^{M_{k}} f_{n}^{2}\left(X_{k, m}\right)-\frac{1}{M_{k}^{3}}\left[\sum_{m=1}^{M_{k}} f_{n}\left(X_{k, m}\right)\right]^{2} \text {. }
$$

It follows from Eqs. (24) and (25) that the variance $V\left(p_{n}\right)$ scales as $1 / N$, where $N$ is the total number of measurements.

\section{B. Data antisqueezing}

As we have seen in Sec. II, the efficiency of our witness can be greatly increased if we construct the witness from probabilities of squeezed Fock states. Equivalently, this means that we should antisqueeze the state before we estimate the probabilities $p_{0}$ and $p_{1}$. In the Heisenberg picture, the antisqueezing transformation boils down to a linear rescaling of quadratures,

$$
x=x_{0} e^{s}, \quad p=p_{0} e^{-s} .
$$

This suggests that it may be possible to perform the antisqueezing on the homodyne data without altering the experimental setup. To show this, we express the measured quadrature operator $x_{\theta}=x \cos \theta+p \sin \theta$ in terms of the antisqueezed quadratures $x_{0}$ and $p_{0}$,

$$
x_{\theta}=x_{0} e^{s} \cos \theta+p_{0} e^{-s} \sin \theta .
$$

We can rewrite this expression as follows:

$$
x_{\theta}=g\left(x_{0} \cos \vartheta+p_{0} \sin \vartheta\right)=g \tilde{x}_{\vartheta},
$$

where the new effective phase $\vartheta$ and scaling factor $g$ are given by

$$
\begin{gathered}
\tan \vartheta=e^{-2 s} \tan \theta, \\
g=\sqrt{e^{2 s} \cos ^{2} \theta+e^{-2 s} \sin ^{2} \theta} .
\end{gathered}
$$

According to the above formulas, the measurement of $x_{\theta}$ can be equivalently interpreted as a measurement of the quadrature $\tilde{x}_{\vartheta}$ of the antisqueezed state, where $\tilde{x}_{\vartheta}=x_{\theta} / g$. Photon number distribution $p_{n}(s)$ of the antisqueezed state can be inferred by a modified formula (21),

$$
p_{n}(s)=\frac{1}{\pi} \int_{0}^{\pi} \int_{-\infty}^{\infty} \frac{1}{g^{2}} w\left(x_{\theta} ; \theta\right) f_{n}\left(\frac{x_{\theta}}{g}\right) d x_{\theta} d \theta .
$$

The factor

$$
\frac{1}{g^{2}}=\frac{d \vartheta}{d \theta}
$$

attributes different weights to the pattern function according to the value of $\theta$ because a homogeneous sampling over $\theta$ is equivalent to an inhomogeneous sampling over $\vartheta$ due to the nontrivial dependence of $\vartheta$ on $\theta$. Looking more carefully at Eq. (32), we can identify the effective pattern functions for reconstruction of diagonal density matrix elements in the basis of squeezed Fock states,

$$
f_{n}\left(x_{\theta}, \theta ; s\right)=\frac{1}{g^{2}} f_{n}\left(\frac{x_{\theta}}{g}\right) .
$$

Note that these generalized pattern functions explicitly depend on $\theta$ through $g$.

\section{Maximum likelihood estimation}

For comparison, we also perform a maximum likelihood estimation [31-33] of the photon number distribution $p_{n}$. For this purpose, we construct a quadrature histogram corresponding to averaged quadrature statistics,

$$
\bar{w}(x)=\frac{1}{\pi} \int_{0}^{\pi} w(x ; \theta) d \theta .
$$

We divide the real axis into equidistant bins with width $\Delta x$. To each measured value $X_{k, m}$ we assign an index $j$ of the corresponding quadrature bin, $j=\operatorname{round}\left(X_{k, m} / \Delta x\right)$, and we increase the counter for this bin by $1 / M_{k}, C_{j} \rightarrow C_{j}+$ $1 / M_{k}$, where initially $C_{j}=0$. The resulting values $C_{j}$ are 
proportional to the probabilities that the quadrature outcome falls into the $j$ th window, $P_{j}=\int_{\left(j-\frac{1}{2}\right) \Delta x}^{\left(j+\frac{1}{2}\right) \Delta x} \bar{w}(x) d x$. We can associate a POVM element $\Pi_{j}$ with each bin, $P_{j}=\operatorname{Tr}\left[\Pi_{j} \rho\right]$. Due to the phase averaging, the POVM elements are diagonal in the Fock state basis, $\Pi_{j}=\sum_{n=0}^{\infty} \Pi_{j, n}|n\rangle\langle n|, \Pi_{j, n} \geqslant 0$, and $\sum_{j} \Pi_{j, n}=1$. The probability of obtaining an outcome in the $j$ th bin is given by $P_{j}=\sum_{n} \Pi_{j, n} p_{n}$ and the likelihood function reads

$$
L=\prod_{j}\left(\sum_{n} p_{n} \Pi_{j, n}\right)^{C_{j}} .
$$

The maximum likelihood estimates $p_{n}$ which maximize the likelihood function $L$ can be numerically determined by an expectation-maximization algorithm $[32,42,43]$.

We can generalize the above procedure to an estimation of $p_{n}$ of the antisqueezed states. According to the formula (29), we need to properly rescale each measurement outcome, hence the bin index is now given by $j=\operatorname{round}\left[X_{k, m} /(g \Delta x)\right]$. Also, we must take into account the weight factor $\frac{d \vartheta}{d \theta}=g^{-2}$ when building the phase-averaged histogram, $C_{j} \rightarrow C_{j}+$ $1 /\left(g^{2} M_{k}\right)$. In this way, we obtain a quadrature histogram that corresponds to the phase-averaged quadrature statistics of the antisqueezed state. Once the histogram is calculated, the probabilities $p_{n}(s)$ can be determined by the expectationmaximization algorithm similarly as before.

\section{RESULTS AND DISCUSSION}

We have estimated the probabilities $p_{0}(s)$ and $p_{1}(s)$ from the experimental data using the pattern functions described in the preceding section. The results are shown in Fig. 6, where the probability pairs $p_{0}(s), p_{1}(s)$ are depicted for $s \in[0,0.4]$. The graph suggests that the state is both nonclassical and quantum non-Gaussian. However, the statistical errors are significant due to a relatively low number of quadrature samples $N=8000$ which leads to standard deviations of the order of $1 / \sqrt{N} \approx 0.011$.

Since there could be statistical correlations between estimates of $p_{0}(s)$ and $p_{1}(s)$, a proper evaluation of the

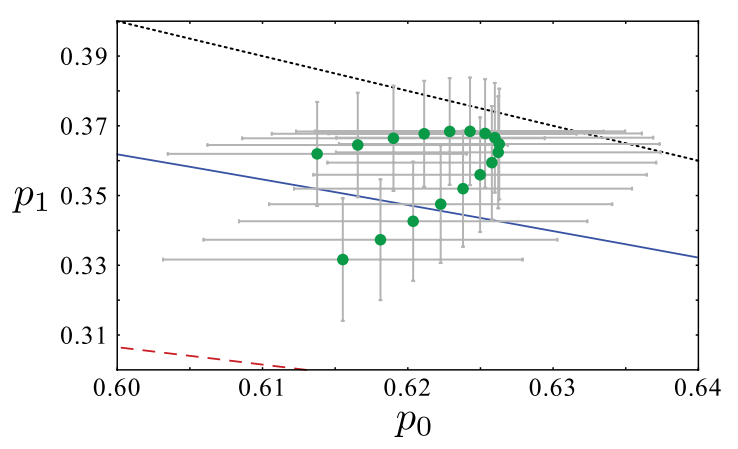

FIG. 6. (Color online) Probability pairs $p_{0}(s)$ and $p_{1}(s)$ estimated from experimental data with the use of pattern functions (green circles). Error bars indicate statistical errors (one standard deviation). The blue solid line represents the boundary above which the state is recognized as quantum non-Gaussian. The red dashed line indicates the nonclassicality boundary and the dotted black line is the ultimate physical boundary $p_{0}+p_{1}=1$.
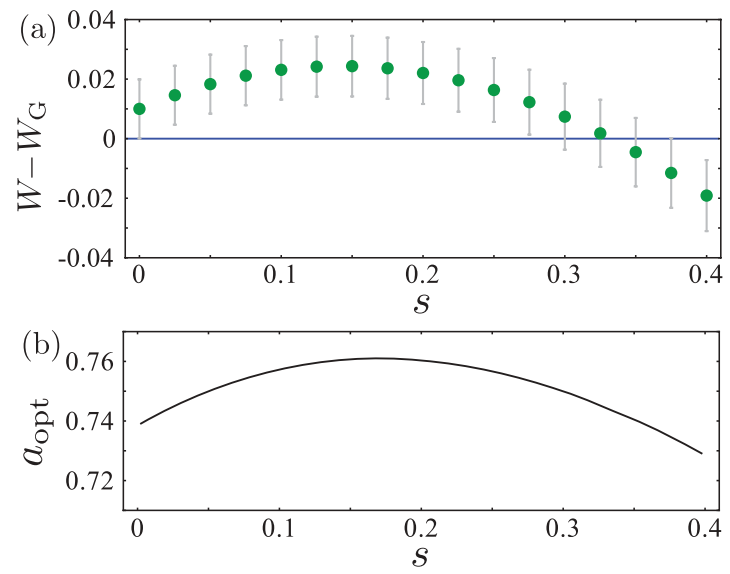

FIG. 7. (Color online) (a) The optimal witness $W\left(a_{\mathrm{opt}}, s\right)-$ $W_{G}\left(a_{\text {opt }}\right)$ and (b) the optimal witness parameter $a_{\text {opt }}$ are plotted as functions of the antisqueezing parameter $s$. The error bars represent one standard deviation.

statistical significance of the observed non-Gaussian character requires determination of statistical error of the witness $W(a, s)$. This is accomplished by calculating the statistical error for the sampling function $f_{W}$ as discussed in Sec. V A. We introduce a normalized relative witness

$$
W_{R}(a, s)=\frac{W(a, s)-W_{G}(a)}{\Delta W(a, s)},
$$

where $\Delta W(a, s)$ is the standard deviation of $W(a, s)$. For each antisqueezing $s$, we maximize $W_{R}$ over $a$. The resulting optimal witnesses are plotted in Fig. 7(a) and the dependence of the optimal value of $a$ on $s$ is shown in Fig. 7(b). Without antisqueezing, the confirmation of non-Gaussian character is not statistically significant, but for the optimal value of antisqueezing the quantum non-Gaussian character is confirmed with much higher confidence. The optimum squeezing yielding the maximum relative witness is $s_{\mathrm{opt}}=0.15$ and we have

$$
W\left(a_{\mathrm{opt}}, s_{\mathrm{opt}}\right)-W_{G}\left(a_{\mathrm{opt}}\right)=0.024 \pm 0.010,
$$

hence the Gaussian threshold is exceeded by 2.4 standard deviations. Since $p_{0}>\frac{1}{2}$, the Wigner function of the state is positive at the origin, where we expect it to be most negative for this kind of state. The strongly nonclassical character of the state indicated by the witness thus could not be uncovered by looking at the negativity of the Wigner function.

Next we compare the experimental results with the theoretical model developed in Sec. IV. We optimized the model parameters to obtain the best fit to the experimental data shown in Fig. 6. In the numerical optimization, we fixed the reflectance of the tap-off beam splitter, $R=1-T=0.077$, and the homodyne detection efficiency $\eta_{H}=0.80$, which were both reliably determined by independent measurements. We also fixed the single-photon efficiency $\eta=0.08$. Although $\eta$ is estimated with a large uncertainty, its value mainly influences the success probability of the experiment while the shape of the theoretical fit remains practically unchanged when $\eta$ varies within the uncertainty interval. The fitting yields the following parameters: $V_{x}=0.364, V_{p}=0.705, Q=0.625$, and $n_{\text {th }}=0$. This theoretical fit is compared with experimental data in Fig. 8 and we achieve very good agreement between 

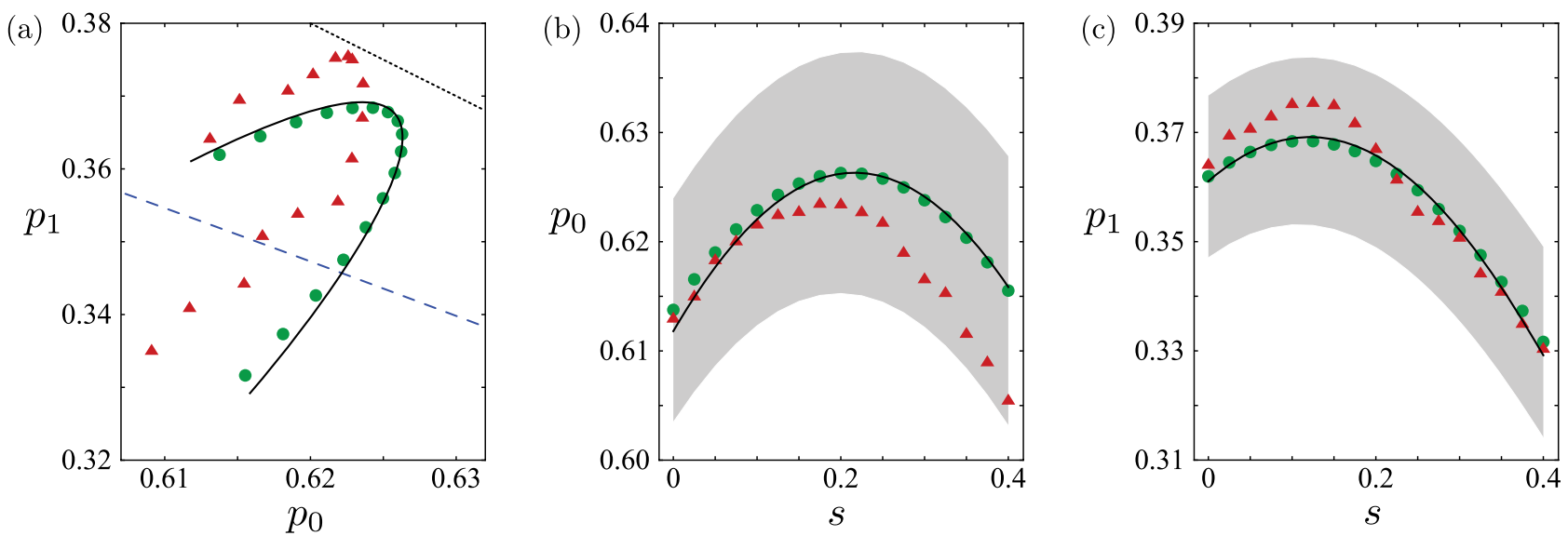

FIG. 8. (Color online) Theoretical fit (solid black line) to the probabilities $p_{0}(s)$ and $p_{1}(s)$ estimated from experimental data using pattern functions (green circles). Panel (a) depicts the probability pairs in the $p_{0}, p_{1}$ plane, while panels (b) and (c) show the dependence of $p_{0}(s)$ and $p_{1}(s)$ on $s$. The gray areas in panels (b) and (c) indicate one standard deviation of estimates obtained by pattern functions. The red triangles represent the results of maximum likelihood estimation of $p_{0}(s)$ and $p_{1}(s)$ from the experimental data. The blue dashed line in panel (a) indicates the maximum $p_{1}$ achievable by Gaussian states and their mixtures for a given $p_{0}$, while the dotted black line marks the ultimate physical boundary $p_{0}+p_{1}=1$.

theory and experiment. For comparison, we also plot in Fig. 8 the results of maximum likelihood estimation of $p_{0}(s)$ and $p_{1}(s)$. The ML estimates agree qualitatively with the results of linear reconstruction. The maximum likelihood method provides slightly higher estimates of $p_{1}$ and slightly lower estimates of $p_{0}$ than linear reconstruction, but the difference is less than one standard deviation; cf. Figs. 8(b) and 8(c). Note also that the ML estimates exhibit a more irregular pattern than the linear estimates based on pattern functions; cf. Fig. 8(a). This is likely caused by the need to construct from the experimental data a quadrature histogram that corresponds to the phase-averaged quadrature statistics of an antisqueezed state. As discussed in Sec. V C, this latter procedure involves rescaling of the data and their relative weights in the histogram. The ratio between maximum and minimum weights reads $e^{4 s}$, hence it grows fast with increasing antisqueezing $s$. This procedure, therefore, tends to amplify any statistical fluctuations in the data, which influences in a nontrivial way the outcome of nonlinear maximum likelihood estimation.

\section{CONCLUSIONS}

In summary, we have experimentally demonstrated the quantum non-Gaussian character of a noisy single-photon squeezed state generated by conditional photon subtraction from pulsed squeezed vacuum. Our approach, which is based on a witness constructed from probabilities of squeezed vacuum and single-photon states, allowed us to reveal the quantum non-Gaussian character of the state with reasonably high statistical confidence. This was achieved by an optimization of the variable squeezing parameter $s$. The generalized witness $W(a, s)$ can identify a much broader class of quantum
non-Gaussian states than the original witness $W(a)$ based on the photon number probabilities $p_{0}$ and $p_{1}[3,4]$ because optimization over various Gaussian operations allows one to suitably match the witness to a given state. We emphasize that the witness is able to detect the quantum non-Gaussian character of states with a positive Wigner function.

We have shown that the pattern functions represent an efficient method of estimation of the witness $W(a, s)$ from the experimental homodyne data. Moreover, this approach provides reliable easy-to-calculate estimates of the statistical error bars. The probability $p_{1}(s)$ is an overlap of the measured state $\rho$ with a squeezed single-photon state. Since this latter state contains only odd Fock states in its Fock-state expansion, the probability $p_{1}(s)$ provides a lower bound on the negativity of the Wigner function at the origin of phase space. In particular, if $p_{1}(s)>0.5$ for any $s$, then the Wigner function is negative at the origin. The pattern functions derived in the present work thus also provide a useful tool for probing the negativity of the Wigner function without the need for a full reconstruction of photon number distribution. A detailed analysis of this will be the subject of a future work.

\section{ACKNOWLEDGMENTS}

This work was supported by the Czech Science Foundation (P205/12/0577) and the Danish Research Agency (Project No. FNU 09-072623). M.J. also acknowledges support from the Operational Program Education for Competitiveness of the European Social Fund (Project No. CZ.1.07/2.3.00/20.0060) of the Ministry of Education, Youth and Sports of the Czech Republic.
[1] R. Glauber, Quantum Theory of Optical Coherence (Wiley-VCH, Weinheim, 2007).

[2] R. L. Hudson, Rep. Math. Phys. 6, 249 (1974).
[3] R. Filip and L. Mišta, Jr., Phys. Rev. Lett. 106, 200401 (2011).

[4] M. Ježek, I. Straka, M. Mičuda, M. Dušek, J. Fiurášek, and R. Filip, Phys. Rev. Lett. 107, 213602 (2011). 
[5] J. Eisert, S. Scheel, and M. B. Plenio, Phys. Rev. Lett. 89, 137903 (2002).

[6] G. Giedke and J. I. Cirac, Phys. Rev. A 66, 032316 (2002).

[7] J. Fiurášek, Phys. Rev. Lett. 89, 137904 (2002).

[8] J. Niset, J. Fiurášek, and N. J. Cerf, Phys. Rev. Lett. 102, 120501 (2009).

[9] S. D. Bartlett, B. C. Sanders, S. L. Braunstein, and K. Nemoto, Phys. Rev. Lett. 88, 097904 (2002).

[10] S. D. Bartlett and B. C. Sanders, Phys. Rev. Lett. 89, 207903 (2002).

[11] M. Ohliger, K. Kieling, and J. Eisert, Phys. Rev. A 82, 042336 (2010).

[12] S. L. Braunstein and P. van Loock, Rev. Mod. Phys. 77, 513 (2005).

[13] Quantum Information with Continuous Variables of Atoms and Light, edited by N. Cerf, G. Leuchs, and E. Polzik (Imperial College Press, London, 2007).

[14] T. Richter and W. Vogel, Phys. Rev. Lett. 89, 283601 (2002).

[15] D. T. Smithey, M. Beck, M. G. Raymer, and A. Faridani, Phys. Rev. Lett. 70, 1244 (1993).

[16] U. Leonhardt, Measuring the Quantum State of Light (Cambridge University Press, Cambridge, 1997).

[17] D.-G. Welsch, W. Vogel, and T. Opatrný, Homodyne Detection and Quantum-State Reconstruction, Vol. 39 of Progress in Optics, edited by E. Wolf (Elsevier, Amsterdam, 1999).

[18] J. Wenger, R. Tualle-Brouri, and P. Grangier, Phys. Rev. Lett. 92, 153601 (2004).

[19] A. Ourjoumtsev, R. Tualle-Brouri, J. Laurat, and Ph. Grangier, Science 312, 83 (2006).

[20] J. S. Neergaard-Nielsen, B. M. Nielsen, C. Hettich, K. Molmer, and E. S. Polzik, Phys. Rev. Lett. 97, 083604 (2006).

[21] K. Wakui, H. Takahashi, A. Furusawa, and M. Sasaki, Opt. Express 15, 3568 (2007).

[22] H. Takahashi, K. Wakui, S. Suzuki, M. Takeoka, K. Hayasaka, A. Furusawa, and M. Sasaki, Phys. Rev. Lett. 101, 233605 (2008).
[23] T. Gerrits, S. Glancy, T. S. Clement, B. Calkins, A. E. Lita, A. J. Miller, A. L. Migdall, S. W. Nam, R. P. Mirin, and E. Knill, Phys. Rev. A 82, 031802(R) (2010).

[24] J. S. Neergaard Nielsen, M. Takeuchi, K. Wakui, H. Takahashi, K. Hayasaka, M. Takeoka, and M. Sasaki, Phys. Rev. Lett. 105, 053602 (2010).

[25] A. Tipsmark, R. Dong, A. Laghaout, P. Marek, M. Ježek, and U. L. Andersen, Phys. Rev. A 84, 050301(R) (2011).

[26] G. M. D'Ariano, U. Leonhardt, and H. Paul, Phys. Rev. A 52, R1801 (1995).

[27] U. Leonhardt, H. Paul, and G. M. D’Ariano, Phys. Rev. A 52, 4899 (1995).

[28] U. Leonhardt, M. Munroe, T. Kiss, Th. Richter, and M. G. Raymer, Opt. Commun. 127, 144 (1995).

[29] Th. Richter, Phys. Lett. A 211, 31 (1996).

[30] Th. Richter, Phys. Rev. A 61, 063819 (2000).

[31] Z. Hradil, Phys. Rev. A 55, R1561 (1997).

[32] J. Řeháček, Z. Hradil, and M. Ježek, Phys. Rev. A 63, 040303 (2001).

[33] A. I. Lvovsky, J. Opt. B 6, S556 (2004).

[34] If $a \geqslant 1$, then $a p_{0}+p_{1}$ is trivially maximized by a Gaussian vacuum state with $p_{0}=1$ and the witness becomes useless.

[35] L. Lachman and R. Filip (unpublished).

[36] D. Menzies and R. Filip, Phys. Rev. A 79, 012313 (2009).

[37] J. Fiurášek, R. García-Patrón, and N. J. Cerf, Phys. Rev. A 72, 033822 (2005).

[38] M. Sasaki and S. Suzuki, Phys. Rev. A 73, 043807 (2006).

[39] R. Tualle-Brouri, A. Ourjoumtsev, A. Dantan, P. Grangier, M. Wubs, and A. S. Sørensen, Phys. Rev. A 80, 013806 (2009).

[40] C. Weedbrook, S. Pirandola, R. García-Patrón, N. J. Cerf, T. C. Ralph, J. H. Shapiro, and S. Lloyd, Rev. Mod. Phys. 84, 621 (2012).

[41] G. M. D'Ariano and M. G. A. Paris, Phys. Rev. A 60, 518 (1999).

[42] A. P. Dempster, N. M. Laird, and D. B. Rubin, J. R. Stat. Soc. B 39, 1 (1977).

[43] Y. Vardi and D. Lee, J. R. Stat. Soc. B 55, 569 (1993). 